\title{
Eliciting Probabilities, Means, Medians, Variances and Covariances without Assuming Risk Neutrality
}

\author{
Karl H. Schlag ${ }^{1}$, Joël J. van der Weele ${ }^{2}$ \\ ${ }^{1}$ University Vienna, Vienna, Austria \\ ${ }^{2}$ Department of Economics, J. W. Goethe University, Frankfurt am Main, Germany \\ Email: karl.schlag@univie.ac.at, vanderweele@econ.uni-frankfurt.com
}

Received November 27, 2012; revised December 29, 2012; accepted January 30, 2013

\begin{abstract}
We are interested in incentivizing experimental subjects to report their beliefs truthfully, without imposing assumptions on their risk preferences. We prove that if subjects are not risk neutral, it is not possible to elicit subjective probabilities or the mean of a subjective probability distribution truthfully using deterministic payments schemes, which are predominant in the literature. We present a simple randomization trick that transforms deterministic rewards into randomized rewards, such that agents with arbitrary risk preferences report as if they were risk neutral. Using this trick, we show how to elicit probabilities, means, medians, variances and covariances of the underlying distribution without assuming risk neutrality.
\end{abstract}

Keywords: Belief Elicitation; Proper Scoring Rules; Lottery Tickets; Risk Preferences

\section{Introduction}

The economic literature on the elicitation of an expert's subjective beliefs has focused on so-called proper scoring rules. These mechanisms, which are used in many economic experiments, reward the expert on the basis of post-elicitation events such that it is in the expert's interest to report her true beliefs if she is risk neutral. The quadratic scoring rule (QSR) [1] is the most popular rule, used to elicit the probability of an event or the mean of a random variable. In the absence of risk neutrality, there is an incentive to report conservative beliefs in order to avoid large losses [2]. This is a problem, since risk neutrality is shown to be widely violated in experimental studies [e.g. 3]. Indeed, Armantier and Treich [4] show experimentally that consistent with risk aversion, elicitation with the quadratic scoring rule leads to conservative bias in reported beliefs.

There are different ways to get around this problem. Offerman et al. [5] propose a way to correct for deviations of risk neutrality and expected utility, by quantifying the size of deviations for each individual. Alternatively, an earlier literature starting with Smith [6] ${ }^{1}$ shows how one can induce risk neutrality by rewarding subjects using binary lottery tickets. This idea has been

\footnotetext{
${ }^{1}$ Smith [6, p. 13] writes that the idea is "adapted from Savage [7]". However, Savage [8, p. 785] 1971 clearly attributes the idea to Smith [6]. Roth and Malouf [9] were the first to induce risk neutrality in this way in an experiment.
}

used to show how to elicit the subjective probability of an event [e.g. 10,11] in a way similar to the elicitation of a reservation price [12].

We extend this literature in several ways. First, we prove that deterministic schemes are not adequate if one does not know the risk preferences of the expert. Second, we combine the literature on scoring rules for risk neutral preferences with the literature on incentivizing with lottery tickets to show that one can elicit a median or any quantile without making assumptions on risk preferences. We also present an alternative way to elicit a probability or mean based on the randomized quadratic scoring rule. Third, we present a new deterministic rule, and its randomized counterpart, to elicit variances and covariances when two independent observations are available.

\section{Preliminaries}

We consider two people, an expert and an elicitor. The expert has subjective beliefs about the distribution $F_{X}$ of a bounded random variable $X$ that yields outcomes belonging to $\Omega \subseteq[a, b]$ with $a<b$. The expert maximizes expected utility for some utility function $u$ on $\mathbb{R}$ such that $u(M)>u(0)$ for some $M$. The elicitor only knows that $X$ yields outcomes belonging to $\Omega$, and would like to learn some parameter $\pi(X) \in \Pi$ of the distribution $F_{X}$. We consider the use of a reward system or scoring rule $g: \Pi \times \Omega \rightarrow \Delta \mathbb{R}$ which rewards the expert on the basis of her report $z \in \Pi$ and a single 
random realization $x$ of $X$. Here, $\Delta \mathbb{R}$ is a distribution over the rewards which includes a deterministic reward as a special case. In the literature, $g(z, x)$ is called strictly proper for $\pi(X)$ if

$E g(\pi(X), x)>E g(z, x)$ for all $z \neq \pi(X)$. We say that a rule elicits $\pi(X)$ if $E u(g(\pi(X), x))>E u(g(z, x))$ for all $z \neq \pi(X)$ and all $u$ with $u(M)>u(0)$.

\section{Limitations of Deterministic Rewards}

Consider an elicitor who wishes either to learn about the mean of some random variable $X$ with support in $\Omega$, or about probability of some event $C \subset \Omega$. We obtain the following result.

Proposition 1. A scoring rule with a deterministic reward cannot elicit the probability $\operatorname{Pr}(X \in C)$ or the mean $E X$.

The proof is in the Appendix. The intuition is simple. The elicitor has only one parameter, the realization $x$, to incentivize the expert to tell the truth. On the other hand, there are two dimensions of uncertainty as the elicitor does not know $\pi(X)$ and $u$.

\section{Probabilistic Elicitation}

We now consider elicitation using probabilistic or randomized reward functions. The idea, first elaborated by Smith [6], is that one pays the experts in lottery tickets rather than money. The size of the prize is given by the probability of winning the lottery. Hence $g(z, x) \in \Delta \mathbb{R}$ where $g(z, x)$ is now the payoff distribution awarded conditional on $(z, x)$. Using this idea, we show how to elicit probabilities, means, different quantiles, and variances and covariances.

\subsection{Randomization Trick}

We use the following "randomization trick" to transform deterministic into probabilistic payoffs. First, given a deterministic reward function $g$, determine $A$ and $B$ such that $A=\min _{z, x} g(z, x)$ and $B=\max _{z, x} g(z, x)$. Second, draw a realization $y$ from a uniform distribution on $[A, B]$ and then pay $M$ if $y \leq g(z, x)$ and pay 0 if $y>g(z, x)$.

Formally, we replace the deterministic reward $g(z, x)$ by the randomized reward

$$
\tilde{g}(z, x)=l\left(M, 0 ; \frac{g(z, x)-A}{B-A}\right),
$$

where $l(M, 0 ; p)$ is a lottery that pays $M$ with probality $p$ and 0 with probability $1-p$. Consequently,

$$
\begin{aligned}
E u(\tilde{g}(z, x))= & \frac{g(z, x)-A}{B-A} u(M)+\frac{B-g(z, x)}{B-A} u(0) \\
& =g(z, x) \frac{u(M)-u(0)}{B-A} \\
& +\frac{B \cdot u(0)-A \cdot u(M)}{B-A}
\end{aligned}
$$

The expected utility of the expert equals an affine transformation of $g(z, x)$. Thus, a report that maximizes her expected utility is a report that maximizes the utility of a risk neutral expert and vice versa. In particular, $\tilde{g}(z, x)$ elicits $\pi(x)$ iff $g(z, x)$ is strictly proper for $\pi(X))^{2}$

\subsection{Eliciting Probabilities}

Randomized rewards for the elicitation of probabilities have received quite some attention. Grether [10] (see also Holt [15, ch. 30] and Karni [16]) presents a simple reward function where a prize is rewarded with some probability that depends on the draw of two uniformly distributed random variables. Allen [11] presents an alternative rule that relies on a draw of a random variable that has a more complex probability distribution. Mclvey and Page [17] uses a randomized version of the quadratic scoring rule in an experimental application, which is similar to the rule we present below.

The QSR (for the event $X \in C$ ) is given by

$$
g_{Q S R}(z, x)=-\left(z-\mathbf{I}_{\{x \in C\}}\right)^{2}
$$

and is strictly proper for $\operatorname{Pr}(X \in C)$ [1]. The randomized quadratic scoring rule (for the event $X \in C$ ), short rQSR, is defined by

$\tilde{g}_{Q S R}(z, x)=l\left(M, 0 ; 1+g_{Q S R}(z, x)\right)$. The following result obtains:

Proposition 2. The randomized quadratic scoring rule elicits $\operatorname{Pr}(X \in C)$.

Note that the expected payoffs under rQSR are identical to those under the rules of Allen [11] and McKelvey and Page [17] when $M=1$.

\subsection{Eliciting the Mean}

To elicit the mean, we combine the randomization trick with the fact that the QSR $g_{Q S R}(z, x)=-(z-x)^{2}$ is a strictly proper scoring rule for the mean (for risk neutral experts). Given $A=0$ and $B=(b-a)^{2}$ we obtain the randomized quadratic scoring rule as defined by

\footnotetext{
${ }^{2}$ Other authors have independently worked on similar mechanisms. Hossain and Okui [13] presents a randomized mechanism for eliciting the mean of a symmetric distribution, allowing for unbounded support with some additional restrictions. Harrison et al. [14] considers a version of the rQSR (see below), which they call the Binary Lottery Procedure. Both papers also consider non-expected utility theory.
} 


$$
\tilde{g}_{Q S R}(z, x)=l\left(M, 0 ; 1+\frac{g_{Q S R}(z, x)}{(b-a)^{2}}\right) .
$$

Proposition 3. The randomized quadratic scoring rule elicits EX.

\subsection{Median and Quantiles}

The quantile scoring rule, due to Cervera and Munoz [18] is a strictly proper scoring rule for the quantile $\alpha$ of the distribution $F_{X}$ of $X$ for any given $\alpha \in(0,1)$. Its reward function is given by $g_{\alpha}(z, x)=\alpha z-(z-x) \mathbf{I}_{z \geq x}$. The randomized quantile scoring rule is hence given by $\tilde{g}_{\alpha}(z, x)=l\left(M, 0 ; \bar{g}_{\alpha}(z, x)\right)$ where

$$
\bar{g}_{\alpha}(z, x)=\frac{\alpha z-(z-x) \mathbf{I}_{z \geq x}+b(1-\alpha)-a}{b-a} .
$$

Proposition 4. The randomized quantile scoring rule elicits the quantile $\alpha$.

In particular, Proposition 4 shows how to elicit the median by setting $\alpha=1 / 2$.

\subsection{Variance and Covariance}

In order to elicit the variance of $F_{X}$ we assume the elicitor can condition on two independent realizations $x_{1}$ and $x_{2}$ of $X$ when rewarding the expert. So we conder a reward function $g\left(z, x_{1}, x_{2}\right)$. We first construct a strictly proper scoring rule. Following Walsh (1962),

$\operatorname{Var} X=E\left(\frac{1}{2}\left(X_{1}-X_{2}\right)^{2}\right)$ where $X_{1}$ and $X_{2}$ are indendent copies of $X$. We combine this with the quadratic scoring rule to obtain that the variance scoring rule $g_{V}\left(z, x_{1}, x_{2}\right)=-\left(z-\frac{1}{2}\left(x_{1}-x_{2}\right)^{2}\right)^{2}$ that is strictly proper for $\operatorname{Var} X$. Given

$$
\bar{g}_{V}\left(z, x_{1}, x_{2}\right)=\frac{g_{V}\left(z, x_{1}, x_{2}\right)+\frac{1}{4}(b-a)^{4}}{\frac{1}{4}(b-a)^{4}}
$$

we obtain the randomized variance scoring rule by

$$
\tilde{g}_{V}\left(z, x_{1}, x_{2}\right)=l\left(M, 0 ; \bar{g}_{V}\left(z, x_{1}, x_{2}\right)\right) .
$$

Proposition 5. The randomized variance scoring rule elicits the variance of $X$.

Similarly we can elicit the covariance given two ranm variables $X_{1}$ and $X_{2}$. We assume that $X_{i} \in\left[a_{i}, b_{i}\right]$ for $i=1,2$. Here we condition on a realization $\left(x_{1}, x_{2}\right)$ drawn from $\left(X_{1}, X_{2}\right)$. Again following Walsh (1962), we use the fact that $\operatorname{Cov}\left(X_{1}, X_{2}\right)=E\left(\frac{1}{2}\left(X_{1}-X_{2}\right)^{2}\right)$ and then use the QSR to define the covariance scoring rule $g_{C}\left(z, x_{1}, x_{2}\right)=-\left(z-\frac{1}{2}\left(x_{1}-x_{2}\right)^{2}\right)^{2}$ that is strictly proper for $\operatorname{Cov}\left(X_{1}, X_{2}\right)$. Given

$$
\begin{gathered}
c=\frac{1}{4}\left(b_{1}-a_{1}\right)\left(b_{2}-a_{2}\right)+\frac{1}{2} \max \left\{b_{2}-a_{1}, b_{1}-a_{2}\right\} \text { and } \\
\bar{g}_{C}\left(z, x_{1}, x_{2}\right)=\frac{g_{C}\left(z, x_{1}, x_{2}\right)+c^{2}}{c^{2}}
\end{gathered}
$$

we obtain the randomized covariance scoring rule by

$$
\tilde{g}_{C}\left(z, x_{1}, x_{2}\right)=l\left(M, 0 ; \bar{g}_{C}\left(z, x_{1}, x_{2}\right)\right) .
$$

Proposition 6. The randomized covariance scoring rule elicits the covariance of $X_{1}$ and $X_{2}$.

\section{Conclusions}

We have rigorously shown the limits of deterministic scoring rules for belief elicitation. To overcome those limitations, we applied the idea of paying in lottery tickets to transform known deterministic scoring rules for belief elicitation, such as the well-known QSR, into randomized rules. These rules provide agents with incentives to truthfully report parameters of a subjective probability distribution for all risk preferences, and can be used in experimental applications.

This paper has considered the theoretical side. On the empirical side, it is an open question whether these rules have the desired properties in actual applications, and how they are best presented to subjects. Selten et al. [19, see also review therein] raises doubt whether subjects rewarded using lotteries behave as if risk neutral in experiments. More recently, Harrison et al. [14,20], and Hossain and Okui [13] provide evidence that the produre can induce subjects to behave more in line with risk neutrality.

\section{REFERENCES}

[1] G. W. Brier, "Verification of Forecasts Expressed in Terms of Probability," Monthly Weather Review, Vol. 78, No. 1, 1950, pp. 1-3. doi:10.1175/1520-0493(1950)078<0001:VOFEIT>2.0.C $\underline{\mathrm{O} ; 2}$

[2] R. Winkler and A. Murphy, "Nonlinear Utility and the Probability Score,” Journal of Applied Meteorology, Vol. 9, 1970, pp. 143-148. doi:10.1175/1520-0450(1970)009<0143:NUATPS $>2.0 . C$ $\underline{\mathrm{O} ; 2}$

[3] C. A. Holt and S. Laury, "Risk Aversion and Incentive Effects,” The American Economic Review, Vol. 92, No. 5, 2002, p. 1644. doi:10.1257/000282802762024700

[4] O. Armantier and N. Treich, "Eliciting Beliefs: Proper Scoring Rules, Incentives, Stakes and Hedging," IDEI Working Paper, Vol. 643, 2010. 
[5] T. Offerman, J. Sonnemans, G. Van de Kuilen and P. P. Wakker, “A Truth Serum for Non-Bayesians," Review of Economic Studies, Vol. 76, No. 4, 2009, pp. 1461-1489. doi:10.1111/j.1467-937X.2009.00557.x

[6] C. Smith, "Consistency in Statistical Inference and Decision,” Journal of the Royal Statistical Society. Series B, Vol. 23, No. 1, 1961, pp. 1-37.

[7] L. J. Savage, “The Foundation of Statistics,” Wiley, New York, 1954

[8] L. Savage, "Elicitation of Personal Probabilities and Expectations," Journal of the American Statistical Association, Vol. 66, No. 336, 1971, pp. 783-801. doi:10.1080/01621459.1971.10482346

[9] A. Roth and M. Malouf, "Game-Theoretic Models and the Role of Information in Bargaining," Psychological review, Vol. 86, No. 6, 1979, pp. 574-594. doi:10.1037/0033-295X.86.6.574

[10] D. Grether, "Financial Incentive Effects and Individual Decision-Making,” Working Paper 401, California Institute of Technology, 1981.

[11] F. Allen, "Discovering Personal Probabilities When Utility Functions Are Unknown,” Management Science, Vol. 33, No. 4, 1987, pp. 542-544. doi:10.1287/mnsc.33.4.542

[12] G. Becker, M. DeGroot and J. Marschak, "Measuring Utility by a Single-Response Sequential Method," Behavioral Science, Vol. 9, No. 3, 1964, p. 226. doi:10.1002/bs.3830090304
[13] T. Hossain and R. Okui, “The Binarized Scoring Rule,” Manuscript, 2009.

[14] G. W. Harrison, J. Martffnez-Correa and J. Swarthout, "Eliciting Subjective Probabilities with Binary Lotteries," Manuscript, Georgia State University, Atlanta, 2012.

[15] C. Holt, "Markets, Games and Strategic Behavior,” Pearson/Addison-Wesley, Boston, 2006.

[16] E. Karni, “A Mechanism for Eliciting Probabilities,” Econometrica, Vol. 77, No. 2, 2009, pp. 603-606. doi:10.3982/ECTA7833

[17] R. McKelvey and T. Page, "Public and Private Information: An Experimental Study of Information Pooling," Econometrica: Journal of the Econometric Society, Vol. 58, No. 6, 1990, pp. 1321-1339. doi:10.2307/2938318

[18] J. L. Cervera and J. Munoz, "Proper Scoring Rules for Fractiles,” In: J. O. Berger, A. P. Dawid and A. F. M. Smith, Eds., Bayesian Statistics 5, Oxford University Press, Oxford, 1996, pp. 513-519.

[19] R. Selten, A. Sadrieh and K. Abbink, "Money Does Not Induce Risk Neutral Behavior, but Binary Lotteries Do even Worse,” Theory and Decision, Vol. 46, 1999, pp. 211-249. doi:10.1023/A:1005038628305

[20] G. W. Harrison, J. Martinez-Correa and T. Swarthout, "Inducing Risk Neutral Preferences with Binary Lotteries: A Reconsideration," Journal of Economic Behavior and Organization, 2012, in Press. doi:10.1016/j.jebo.2012.09.008 


\section{Appendix}

Proof of Proposition 1. If one can elicit the mean of a random variable for all distributions in $\Omega$ then one can also elicit the probability of an event as

$\operatorname{Pr}(X \in C)=E Y \quad$ if $\quad Y \quad$ is the Bernoulli random variable such that $Y=1$ if and only if $X \in C$. Hence it is enough to show that one cannot elicit $\operatorname{Pr}(X \in C)$ to prove that one cannot elicit $E X$.

We first show that $g(z, 1)$ and $g(z, 0)$ are differentiable almost everywhere. Once this is established the first order conditions reveal the impossibility.

Consider $X^{\prime} \in\{0,1\}$ where $X^{\prime}=\mathbf{I}_{\{X \in E\}}$. So $E X^{\prime}=\operatorname{Pr}\left(X^{\prime}=1\right)=\operatorname{Pr}(X \in E)$. Let $p=\operatorname{Pr}(X \in E)$.

Assume that $g$ elicits $\operatorname{Pr}(X \in E)$ for all concave $u$. Then we have for all $z$

$$
\begin{gathered}
p u(g(p, 1))+(1-p) u(g(p, 0)) \\
>p u(g(z, 1))+(1-p) u(g(z, 0)) .
\end{gathered}
$$

For $z<p$ and $u=I d$ we have

$$
\begin{aligned}
& p g(p, 1)+(1-p) g(p, 0) \\
& >p g(z, 1)+(1-p) g(z, 0) \\
& =z g(z, 1)+(1-z) g(z, 0)+(p-z)[g(z, 1)-g(z, 0)] \\
& \geq z g(p, 1)+(1-z) g(p, 0)+(p-z)[g(z, 1)-g(z, 0)]
\end{aligned}
$$$$
\text { so }
$$

$$
(p-z)[g(p, 1)-g(p, 0)]>(p-z)[g(z, 1)-g(z, 0)]
$$

so

$$
g(p, 1)-g(p, 0)>g(z, 1)-g(z, 0) .
$$

Hence we have shown that $g(z, 1)-g(z, 0)$ is strictly increasing in $z$.

Similarly, for $z>p$ we have

$$
p(g(p, 1)-g(z, 1))+(1-p)(g(p, 0)-g(z, 0)) \geq 0
$$

and since

$$
g(z, 1)-g(z, 0)>g(p, 1)-g(p, 0)
$$

it follows that $g(p, 0)>g(z, 0)$. So $g(z, 0)$ strictly decreasing in $z$ and hence $g(z, 1)$ is strictly increasing.

From the above two strict monotonicity statements we obtain that $g(z, 1)$ and $g(z, 0)$ are differentiable almost everywhere. Let $D$ be the set where they are differentiable.

For $p \in D$ and differentiable $u$ we can calculate

$$
\begin{aligned}
& \frac{\mathrm{d}}{\mathrm{d} z}(p u(g(z, 1))+(1-p) u(g(z, 0))) \\
= & p u^{\prime}(g(p, 1)) g^{\prime}(z, 1)+(1-p) u^{\prime}(g(p, 0)) g^{\prime}(z, 0)
\end{aligned}
$$

and infer that

$$
\begin{aligned}
& p u^{\prime}(g(p, 1)) g^{\prime}(p, 1) \\
& +(1-p) u^{\prime}(g(p, 0)) g^{\prime}(p, 0)=0 .
\end{aligned}
$$

It is easy to argue with generalized version of the intermediate value theorem that there is $p \in D \backslash\{0\}$ such that $g^{\prime}(p, 1)>0$. Consider $u$ that is differentiable with $u^{\prime}>0$. Then rewrite (1) as:

$$
\frac{u^{\prime}(g(p, 0))}{u^{\prime}(g(p, 1))}=-\frac{(1-p) g^{\prime}(p, 0)}{p g^{\prime}(p, 1)} .
$$

Since $g(z, 1)-g(z, 0)$ is strictly increasing in $z$ there is some $p_{0} \in B \cap(0,1)$ such that $g\left(p_{0}, 1\right) \neq g\left(p_{0}, 0\right)$. So when $p=p_{0}$ the left hand side of (2) depends on $u$. Therefore, (2) cannot hold for all $u$. 